\title{
Hybrid algorithm for an $\alpha$-nonexpansive mapping in a Banach space
}

\author{
Zi-Ming Wang ${ }^{a, *}$, Yongfu Su ${ }^{b}$, Jinlong Kang ${ }^{b}$ \\ ${ }^{a}$ Department of Foundation, Shandong Yingcai University, Jinan 250104, P.R. China. \\ ${ }^{b}$ Department of Mathematics, Tianjin Polytechnic University, Tianjin 300160, P.R. China. \\ ${ }^{c}$ Department of Foundation, Xi'an Communication of Institute, Xi'an 710106, P.R. China. \\ This paper is dedicated to Professor Ljubomir Ćirić \\ Communicated by Professor V. Berinde
}

\begin{abstract}
In this paper, we prove strong convergence theorem by the hybrid method for an $\alpha$-nonexpansive mapping in a Banach space. Our results complement and enrich the research contents of $\alpha$-nonexpansive mapping. Simultaneously, our main result generalizes Takahashi, Takeuchi, Kubota's result[W. Takahashi, Y. Takeuchi , R. Kubota, J. Math. Anal. Appl. 341 (2008) 276-286].C)2012 NGA. All rights reserved.
\end{abstract}

Keywords: $\alpha$-nonexpansive mapping, $\alpha$-mean-asymptotically-nonexpansive mapping, Hybrid algorithm, Fixed point, Banach space.

2010 MSC: Primary 47H05, 47H09; Secondary 47H10.

\section{Introduction}

Let $E$ be a real Banach space and let $C$ be a nonempty closed convex subset of $E$. A mapping $T: C \rightarrow C$ is said to be nonexpansive, if

$$
\|T x-T y\| \leq\|x-y\|, \quad \text { for each } x, y \in C .
$$

Lots of iterative schemes for nonexpansive mappings have been introduced (see [1], 2], [3]), furthermore, many strong convergence theorems for nonexpansive mapping have been proved. On the other hand, there

\footnotetext{
${ }^{*}$ Corresponding author

Email addresses: wangziming1983@yahoo.com.cn (Zi-Ming Wang), suyongfu@tjpu.edu.cn (Yongfu Su), kangjinlong1979@yahoo.cn (Jinlong Kang)
} 
are many nonlinear mappings which are more general than the nonexpansive mapping. Compare to the exist problem of fixed point of those mapping, the iterative methods for finding fixed point is also very useful in studying in the fixed point theory and the theory of equations in other fields.

In 2007, Gobel and Pineda [4 introduced and studied a new mapping, called $\alpha$-nonexpansive mapping. The mapping is more general than the nonexpansive one.

Definition 1.1. For a given multiindex $\alpha=\left(\alpha_{1}, \alpha_{2}, \cdots, \alpha_{n}\right)$ satisfies $\alpha_{i} \geq 0, i=1,2, \cdots, n$ and $\sum_{i=1}^{n} \alpha_{i}=1$. A mapping $T: C \rightarrow C$ is said to be $\alpha$-nonexpansive if

$$
\sum_{i=1}^{n} \alpha_{i}\left\|T^{i} x-T^{i} y\right\| \leq\|x-y\|, \quad \forall x, y \in C .
$$

In order to show that the class of $\alpha$-nonexpansive mapping is more general than the one of nonexpansive mappings, we give an example.

Example 1.2. Let $E=\mathbb{R}^{1}$, and

$$
T(x)=\left\{\begin{array}{lll}
0, & \text { if } & x=0 ; \\
\frac{1}{x}, & \text { if } & x \in(0,+\infty) .
\end{array}\right.
$$

Then, $T$ is not nonexpansive but $\alpha$-nonexpansive.

Proof. Obviously, $T$ is not nonexpansive. Taking $x=\frac{1}{2}, y=0$, by the definition of $T x$, we have

$$
\|T x-T y\|=|2-0|>\left|\frac{1}{2}-0\right|=\|x-y\| .
$$

On the other hand, for every $x, y \in[0,+\infty)$, we have

$$
\left\|T^{2} x-T^{2} y\right\|=\|x-y\| .
$$

Therefore, we can affirm that

$$
0\|T x-T y\|+\left\|T^{2} x-T^{2} y\right\|=\|x-y\|
$$

where $\alpha=\left(\alpha_{1}, \alpha_{2}\right)=(0,1)$. Then $T$ is an $\alpha$-nonexpansive mapping but not a nonexpansive one.

If $T$ is nonexpansive self-mapping, we can imply that $T$ must be $\alpha$-nonexpansive one, where $\alpha=$ $\left(\alpha_{1}, \alpha_{2}, \cdots, \alpha_{n}\right)=\left(\frac{1}{n}, \frac{1}{n}, \cdots, \frac{1}{n},\right)$.

For technical reason we always assume that the first coefficient $\alpha_{1}$ is nonzero, that is, $\alpha_{1}>0$. In this case the mapping $T$ satisfies the Lipschitz condition

$$
\|T x-T y\| \leq \frac{1}{\alpha_{1}}\|x-y\|, \quad \forall x, y \in C .
$$

Recall a mapping $T: C \rightarrow C$ is said to be an asymptotically nonexpansive mapping, if exists a sequence of reals $\left\{\gamma_{n}\right\}$ with $0 \leq \gamma_{n} \rightarrow 0$ such that

$$
\left\|T^{n} x-T^{n} y\right\| \leq\left(1+\gamma_{n}\right)\|x-y\|, \quad \forall x, y \in C .
$$

Noticing that, with regard to $(1.4)$, take $n=1,2, \cdots, n$, we have

$$
\begin{aligned}
& \|T x-T y\| \leq\left(1+\gamma_{1}\right)\|x-y\|, \\
& \left\|T^{2} x-T^{2} y\right\| \leq\left(1+\gamma_{2}\right)\|x-y\|,
\end{aligned}
$$




$$
\left\|T^{n} x-T^{n} y\right\| \leq\left(1+\gamma_{n}\right)\|x-y\| .
$$

By multiplying the above inequalities with $\alpha_{i} \geq 0, i=1,2, \cdots, n$, respectively, we have

$$
\begin{aligned}
& \alpha_{1}\|T x-T y\| \leq \alpha_{1}\left(1+\gamma_{1}\right)\|x-y\|=\alpha_{1}\|x-y\|+\alpha_{1} \gamma_{1}\|x-y\|, \\
& \alpha_{2}\left\|T^{2} x-T^{2} y\right\| \leq \alpha_{2}\left(1+\gamma_{2}\right)\|x-y\|=\alpha_{2}\|x-y\|+\alpha_{2} \gamma_{2}\|x-y\|, \\
& \ldots \\
& \alpha_{n}\left\|T^{n} x-T^{n} y\right\| \leq \alpha_{n}\left(1+\gamma_{n}\right)\|x-y\|=\alpha_{n}\|x-y\|+\alpha_{n} \gamma_{n}\|x-y\| .
\end{aligned}
$$

Adding all the inequalities above, we obtain

$$
\sum_{i=1}^{n} \alpha_{i}\left\|T^{i} x-T^{i} y\right\| \leq \sum_{i=1}^{n} \alpha_{i}\|x-y\|+\sum_{i=1}^{n} \alpha_{i} \gamma_{i}\|x-y\|
$$

Since $\sum_{i=1}^{n} \alpha_{i}=1$ and suppose that $\lim _{n \rightarrow \infty} \sum_{i=1}^{n} \alpha_{i} \gamma_{i}=0$, we have

$$
\sum_{i=1}^{n} \alpha_{i}\left\|T^{i} x-T^{i} y\right\| \leq\left(1+\lambda_{n}\right)\|x-y\|
$$

where $\lambda_{n}=\sum_{i=1}^{n} \alpha_{i} \gamma_{i}$.

Definition 1.3. For a given multiindex $\alpha=\left(\alpha_{1}, \alpha_{2}, \cdots, \alpha_{n}\right)$ satisfies $\alpha_{i} \geq 0, i=1,2, \cdots, n$ and $\sum_{i=1}^{n} \alpha_{i}=1$. A mapping $T: C \rightarrow C$ is said to be $\alpha$-mean-asymptotically-nonexpansive if exists a sequence of reals $\left\{\lambda_{n}\right\}$ with $0 \leq \lambda_{n} \rightarrow 0$ such that

$$
\sum_{i=1}^{n} \alpha_{i}\left\|T^{i} x-T^{i} y\right\| \leq\left(1+\lambda_{n}\right)\|x-y\| .
$$

Remark 1.4. From the analysis above and Definition 1.1, we know that each $\alpha$-nonexpansive mapping is an $\alpha$-mean-asymptotically-nonexpansive one.

The last observation is that (1.2) implies that for $\alpha=\left(\alpha_{1}, \alpha_{2}, \cdots, \alpha_{n}\right)$ the mapping

$$
T_{\alpha} x=\sum_{i=1}^{n} \alpha_{i} T^{i} x, \quad \forall x \in C
$$

is nonexpansive. However, nonexpansiveness of $T_{\alpha}$ is much weaker than (1.2), for instance, it doesn't entail the continuity of $T$ (see [4]).

Recently, Chakkrid Klin-eam, Suthep Suantai [5, introduced the relation of the fixed point sets between $\alpha$-nonexpasive operator and $T_{\alpha}$ operator. they give the following theorem:

Theorem 1.5. (see Theorem 3.1 of Klin-eam, Suantai [5]) Let $C$ be a closed convex subset of a Banach space $E$ and for all $n \in \mathbb{N}$, let $\alpha=\left(\alpha_{1}, \alpha_{2}, \cdots, \alpha_{n}\right)$ such that $\alpha_{i} \geq 0, i=1,2, \cdots, n, \alpha_{1}>0$ and $\sum_{i=1}^{n} \alpha_{i}=1$. Let $T$ be an $\alpha$-nonexpansive mapping from $C$ into itself. If $\alpha_{1}>\frac{1}{\sqrt[n-1]{2}}$, then $F(T)=F\left(T_{\alpha}\right)$, where $F(T)$ is the fixed point set of $T$.

At the same time, they have succeeded in proving the demiclosedness principle for the $\alpha$-nonexpansive mappings. 
Theorem 1.6. (see Theorem 3.4 of Klin-eam, Suantai [5]) Let $C$ be a closed convex subset of a Banach space $E$ and for all $n \in \mathbb{N}$, let $\alpha=\left(\alpha_{1}, \alpha_{2}, \cdots, \alpha_{n}\right)$ such that $\alpha_{i} \geq 0, i=1,2, \cdots, n, \alpha_{1}>0$ and $\sum_{i=1}^{n} \alpha_{i}=1$. Let $T$ be an $\alpha$-nonexpansive mapping from $C$ into itself with $\alpha_{1}>\frac{1}{\sqrt[n-1]{2}}$, if $\left\{x_{n}\right\} \subset C$ converges weakly to $x$ and $\left\{x_{m}-T x_{m}\right\}$ converges strongly to 0 as $n \rightarrow \infty$, then $x \in F(T)$.

Motivated by results above, we prove strong convergence theorems by the hybrid method for an $\alpha$-nonexpansive mapping in a Banach space.

\section{Preliminaries}

In what follows, $E$ denotes a real Banach space with norm $\|\cdot\|$ and $E^{*}$ the dual space of $E$. The norm of $E^{*}$ is also denoted by $\|\cdot\|$. For $x^{*} \in E^{*}$, its value at $x \in E$ is denoted by $\left\langle x, x^{*}\right\rangle$. The normalized duality mapping $J$ from $E$ to $E^{*}$ is defined by

$$
J(x)=\left\{x^{*} \in E^{*}:\left\langle x, x^{*}\right\rangle=\|x\|^{2}=\left\|x^{*}\right\|^{2}\right\}
$$

for all $x \in E$. Some properties of the duality mapping have been given in [6, 7].

A Banach space $E$ is said to be strictly convex if $\|x+y\|<2$ for all $x, y \in E$ with $\|x\|=\|y\|=1$ and $x \neq y$. A Banach space $E$ is also said to be uniformly convex if $\lim _{n \rightarrow \infty}\left\|x_{n}-y_{n}\right\|=0$ for any two sequences $\left\{x_{n}\right\}$ and $\left\{y_{n}\right\}$ in $E$ such that $\left\|x_{n}\right\|=\left\|y_{n}\right\|=1$ and $\lim _{n \rightarrow \infty}\left\|x_{n}+y_{n}\right\|=2$. We also know that if $E$ is a uniformly convex Banach space, then $x_{n} \rightarrow x$ and $\left\|x_{n}\right\| \rightarrow\|x\|$ imply $x_{n} \rightarrow x$. Let $U=\{x \in E:\|x\|=1\}$ be the unit sphere of $E$, then the Banach space $E$ is said to be smooth if

$$
\lim _{n \rightarrow \infty} \frac{\|x+t y\|-\|x\|}{t}
$$

exists for each $x, y \in U$.

Let $C$ be a nonempty closed convex subset of a smooth, strictly convex, and reflexive Banach space $E$. Then, for arbitrarily fixed $x \in E$, a function $C \ni\|y-x\| \in \mathbb{R}$ has a unique minimizer $y_{x} \in C$. Using such a point, we define the metric projection $P_{C}$ by $P_{C} x=y_{x}=\operatorname{argmin}_{y \in C}\|x-y\|^{2}$ for every $x \in E$. In a similar fashion, we can see that a function $C \ni y \mapsto \phi(x, y) \in \mathbb{R}$ also has a unique minimizer $z_{x} \in C$. The generalized projection $\Pi_{C}$ of $E$ onto $C$ is defined by $\Pi_{C} x=z_{x}=\operatorname{argmin}_{y \in C} \phi(x, y)$ for every $x \in E$; see [8].

In fact, we have the following result.

Lemma 2.1. (see [8]) Let $C$ be a nonempty closed and convex subset of a real reflexive, strictly convex, and smooth Banach space $E$ and let $x \in E$. Then, there exists a unique element $x_{0} \in C$ such that $\phi\left(x_{0}, x\right)=$ $\min \{\phi(z, x): z \in C\}$.

In order to prove our results, the following lemmas are needed.

Lemma 2.2. (see [8]) Let $C$ be a nonempty closed and convex subset of a real smooth Banach space E, Let $x \in E$. Then, $x_{0}=\Pi_{C} x$ if and only if

$$
\left\langle z-x_{0}, J x_{0}-J x\right\rangle \geq 0, \quad \forall z \in C .
$$

In Chakkrid Klin-eam, Suthep Suantai [5], they also give the following lemma:

Lemma 2.3. (see Lemma 3.3 of Klin-eam, Suantai [5]) Let $C$ be a closed convex subset of a Banach space $E$ and for all $n \in \mathbb{N}$, let $\alpha=\left(\alpha_{1}, \alpha_{2}, \cdots, \alpha_{n}\right)$ such that $\alpha_{i} \geq 0, i=1,2, \cdots, n, \alpha_{1}>0$ and $\sum_{i=1}^{n} \alpha_{i}=1$. Let $T$ be an $\alpha-$ nonexpansive mapping from $C$ into itself. If $\alpha_{1}>\frac{1}{\sqrt[n-1]{2}}$. Let $\left\{x_{m}\right\}$ be a bounded sequence in $C$, then $\left\|x_{m}-T x_{m}\right\| \rightarrow 0$ if and only if $\left\|x_{m}-T_{\alpha} x_{m}\right\| \rightarrow 0$ as $m \rightarrow \infty$.

A mapping $T: C \rightarrow C$ is said to be closed, if for any sequence $\left\{x_{n}\right\} \subset C$ with $x_{n} \rightarrow x$ and $T x_{n} \rightarrow y$, then $T x=y$. 
Lemma 2.4. Let $C$ be a closed convex subset of a Banach space $E$ and for all $n \in \mathbb{N}$, let $\alpha=\left(\alpha_{1}, \alpha_{2}, \cdots, \alpha_{n}\right)$ such that $\alpha_{i} \geq 0, i=1,2, \cdots, n, \alpha_{1}>0$ and $\sum_{i=1}^{n} \alpha_{i}=1$. Let $T$ be an $\alpha$-nonexpansive mapping from $C$ into itself. If $\alpha_{1}>\frac{1}{\sqrt[n-1]{2}}$. Let $\left\{x_{m}\right\} \subset C$ converges strongly to $x$ and $\left\{x_{n}-T x_{m}\right\}$ converges strongly to 0 as $n \rightarrow \infty$, then $x \in F(T)$.

Proof. Since $\left\{x_{n}-T x_{m}\right\}$ converges strongly to 0 as $n \rightarrow \infty$, from Lemma 2.3, we have $\left\{x_{m}-T_{\alpha} x_{m}\right\}$ converges stronglly to 0 as $n \rightarrow \infty$. Since $T_{\alpha}$ is a nonexpansive mapping, which is closed. We have $x \in F\left(T_{\alpha}\right)$. From Theorem 1.5 , we obtain that $x \in F(T)$.

Lemma 2.5. Let $C$ be a uniformly convex and smooth Banach space $E$ and for all $n \in \mathbb{N}$, let $\alpha=\left(\alpha_{1}, \alpha_{2}, \cdots\right.$ $\left.\cdot, \alpha_{n}\right)$ such that $\alpha_{i} \geq 0, i=1,2, \cdots, n, \alpha_{1}>0$ and $\sum_{i=1}^{n} \alpha_{i}=1$. Let $T$ be an $\alpha$-nonexpansive mapping from $C$ into itself. If $\alpha_{1}>\frac{1}{\sqrt[n-1]{2}}$. Then $F(T)$ is closed and convex.

Proof. By Theorem 1.5, we have $F(T)=F\left(T_{\alpha}\right)$. Since $T_{\alpha}$ is a nonexpansive mapping, we know that $F\left(T_{\alpha}\right)$ is closed and convex here. So, with regard to an $\alpha$-nonexpansive $T$, we also have that $F(T)$ is closed and convex.

\section{The Main Result}

In this section, we consider a strong convergence theorem for an $\alpha$-nonexpansive operator in a Banach space.

Theorem 3.1. Let $C$ be a closed convex subset of a uniformly convex and smooth Banach space E. Let $\alpha=\left(\alpha_{1}, \alpha_{2}, \cdots, \alpha_{n}\right)$ such that $\alpha_{i} \geq 0, i=1,2, \cdots, n, \alpha_{1}>0$ and $\sum_{i=1}^{n} \alpha_{i}=1$, let $T: C \rightarrow C$ be an $\alpha$-nonexpansive mapping such that $F(T) \neq \emptyset$. For $x_{0} \in E, C_{1}=C$ and $x_{1}=\Pi_{C_{1}} x_{0}$, define a sequence $\left\{x_{n}\right\}$ of $C$ as follows:

$$
\left\{\begin{array}{l}
y_{n}=\left(1-\beta_{n}\right) x_{n}+\beta_{n} T x_{n}, \\
C_{n+1}=\left\{z \in C_{n}:\left\|y_{n}-z\right\| \leq\left\|x_{n}-z\right\|\right\}, \\
x_{n+1}=\Pi_{C_{n+1}} x_{0}, \quad n \in \mathbb{N},
\end{array}\right.
$$

where $0<a \leq \beta_{n} \leq 1$, for all $n \in \mathbb{N}$. If $\alpha_{1}>\frac{1}{\sqrt[n-1]{2}}$, Then, $x_{n}$ converges strongly to $z_{0}=\Pi_{F(T)} x_{0}$.

Proof. Firstly, we show that $C_{n}$ is closed and convex and $F(T) \subset C_{n}$ for every $n \in \mathbb{N}$

It is obvious that $C_{n}$ is closed, for every $n \in \mathbb{N}$. It follows that $C_{n}$ is convex for every $n \in \mathbb{N}$. Since

$$
\left\|y_{n}-z\right\| \leq\left\|x_{n}-z\right\|
$$

is equivalent to

$$
\left\|y_{n}-x_{n}\right\|^{2}+2\left\langle y_{n}-x_{n}, J x_{n}-J z\right\rangle \leq 0 .
$$

So, $C_{n}$ is convex for every $n \in \mathbb{N}$. For $u \in F(T)$, we compute that

$$
\begin{aligned}
\left\|y_{n}-u\right\|= & \left\|\left(1-\beta_{n}\right) x_{n}+\beta_{n} T x_{n}-u\right\| \\
\leq & \left(1-\beta_{n}\right)\left\|x_{n}-u\right\|+\beta_{n}\left\|T x_{n}-u\right\| \\
= & \left(1-\beta_{n}\right)\left\|x_{n}-u\right\|+\beta_{n}\left\|T x_{n}-T_{\alpha} u\right\| \\
= & \left(1-\beta_{n}\right)\left\|x_{n}-u\right\|+\beta_{n} \| \alpha_{1}\left(T x_{n}-T u\right) \\
& +\alpha_{2}\left(T x_{n}-T^{2} u\right)+\cdots+\alpha_{n}\left(T x_{n}-T^{n} u\right) \| \\
\leq & \left(1-\beta_{n}\right)\left\|x_{n}-u\right\|+\beta_{n} \frac{1-\alpha_{1}^{n-1}}{\alpha_{1}^{n-1}}\left\|x_{n}-T u\right\| \\
\leq & \left(1-\beta_{n}\right)\left\|x_{n}-u\right\|+\beta_{n}\left\|x_{n}-u\right\| \\
= & \left\|x_{n}-u\right\| .
\end{aligned}
$$


It implies that $u \in C_{n}$, for each $n \in \mathbb{N}$. So, we have $F(T) \subset C_{n}$ for all $n \in \mathbb{N}$.

Next, we prove that $\{x\}$ is bounded. Since $F(T)$ is a nonempty closed convex subset of $C$, there exists a unique element $z_{0} \in F(T)$ such that $z_{0}=\Pi_{F(T)} x_{0}$. From $x_{n+1}=\Pi_{C_{n+1}} x_{0}$, we have

$$
\left\|x_{n+1}-x_{0}\right\| \leq\left\|z-x_{0}\right\|
$$

for every $z \in C_{n+1}$. As $z_{0} \in F(T) \subset C_{n+1}$, we obtain

$$
\left\|x_{n+1}-x_{0}\right\| \leq\left\|z_{0}-x_{0}\right\|,
$$

for every $n \in \mathbb{N}$. This implies that $\left\{x_{n}\right\}$ is bounded.

On the other hand, $C_{n+1} \subset C_{n}$ for all $n \in \mathbb{N}$, we have

$$
x_{n+1}=\Pi_{C_{n+1}} x_{0} \in C_{n+1} \subset C_{n},
$$

for all $n \in \mathbb{N}$. Since $x_{n}=\Pi_{C_{n}} x_{0}$, we obtain that

$$
\left\|x_{n}-x_{0}\right\| \leq\left\|x_{n+1}-x_{0}\right\|,
$$

for all $n \in \mathbb{N}$. It follows from (3.6) that the limit of $\left\{x_{n}-x_{0}\right\}$ exists.

Since $C_{m} \subset C_{n}, x_{m+1}=\Pi_{C_{m+1}} x_{0} \in C_{m} \subset C_{n}$ for all $m \geq n$ and $x_{m+1}=\Pi_{C_{m+1}} x_{0}$, in view of Lemma 2.1, one has

$$
\left\langle x_{n+1}-x_{0}, J x_{n+1}-J x_{n+1}\right\rangle \geq 0 .
$$

We compute that

$$
\begin{aligned}
\left\|x_{m+1}-x_{n+1}\right\|^{2}= & \left\|x_{m+1}-x_{0}-\left(x_{n+1}-x_{0}\right)\right\|^{2} \\
= & \left\|x_{m+1}-x_{0}\right\|^{2}+\left\|x_{n+1}-x_{0}\right\|^{2}-2\left\langle x_{n+1}-x_{0}, J x_{m+1}-J x_{0}\right\rangle \\
= & \left\|x_{m+1}-x_{0}\right\|^{2}+\left\|x_{n+1}-x_{0}\right\|^{2} \\
& -2\left\langle x_{n+1}-x_{0}, J x_{m+1}-J x_{n+1}+J x_{n+1}-J x_{0}\right\rangle \\
= & \left\|x_{m+1}-x_{0}\right\|^{2}-\left\|x_{n+1}-x_{0}\right\|^{2}-2\left\langle x_{n+1}-x_{0}, J x_{m+1}-J x_{n+1}\right\rangle \\
\leq & \left\|x_{m+1}-x_{0}\right\|^{2}-\left\|x_{n+1}-x_{0}\right\|^{2} .
\end{aligned}
$$

Since the limit of $\left\|x_{n+1}-x_{0}\right\|$ exists, we get

$$
\lim _{n \rightarrow \infty}\left\|x_{m}-x_{n}\right\|=0 .
$$

Therefore, $\left\{x_{n}\right\}$ is a Cauchy sequence. Since Banach space is a complete metric space and $C$ is closed and convex, one can assume that

$$
x_{n} \rightarrow p, \quad \text { as } n \rightarrow \infty .
$$

Noticing that $x_{n+1}=\Pi_{C_{n+1}} x_{0} \in C_{n+1}$, we obtain that

$$
\left\|y_{n}-x_{n+1}\right\| \leq\left\|x_{n}-x_{n+1}\right\| .
$$

In view of (3.10), we have that

$$
\left\|y_{n}-x_{n+1}\right\| \rightarrow 0, \quad \text { as } n \rightarrow \infty
$$

and

$$
\left\|y_{n}-x_{n}\right\| \leq\left\|y_{n}-x_{n+1}\right\|+\left\|x_{n+1}-x_{n}\right\| \rightarrow 0, \text { as } n \rightarrow \infty .
$$

From $y_{n}=\left(1-\beta_{n}\right) x_{n}+\beta_{n} T x_{n}$, we have

$$
\left\|x_{n}-T x_{n}\right\|=\frac{1}{\beta_{n}}\left\|y_{n}-x_{n}\right\| .
$$


Because of assumption that $0<a \leq \beta_{n} \leq 1$, we have

$$
\lim _{n \rightarrow \infty}\left\|x_{n}-T x_{n}\right\|=0
$$

This implies from Lemma 2.4 that $p \in F(T)$.

Finally, we prove that $p=z_{0}=\Pi_{F(T)} x_{0}$.

From $x_{n+1}=\Pi_{C_{n+1}} x_{0}$ and Lemma 2.1, we have

$$
\left\langle J x_{0}-J x_{n+1}, x_{n+1}-y\right\rangle \geq 0,
$$

for all $y \in C_{n+1}$. Since $F(T) \subset C_{n+1}$, we also have that

$$
\left\langle J x_{0}-J x_{n+1}, x_{n+1}-q\right\rangle \geq 0,
$$

for all $q \in F(T)$. By taking limit in (3.17), one has

$$
\left\langle J x_{0}-J x_{n+1}, p-q\right\rangle \geq 0,
$$

for all $q \in F(T)$. Now, by Lemma 2.1 again, we have that

$$
p=z_{0}=\Pi_{F(T)} x_{0} .
$$

The proof is completed.

Because every nonexpansive mapping is $\alpha$-nonexpansive, the following corollary can be obtain by Theorem 3.1 .

Corollary 3.2. Let $C$ be a closed convex subset of a uniformly convex and smooth Banach space E. Let $T: C \rightarrow C$ be a nonexpansive mapping such that $F(T) \neq \emptyset$. For $x_{0} \in E, C_{1}=C$ and $x_{1}=\Pi_{C_{1}} x_{0}$, define a sequence $\left\{x_{n}\right\}$ of $C$ as follows:

$$
\left\{\begin{array}{l}
y_{n}=\left(1-\beta_{n}\right) x_{n}+\beta_{n} T x_{n}, \\
C_{n+1}=\left\{z \in C_{n}:\left\|y_{n}-z\right\| \leq\left\|x_{n}-z\right\|\right\}, \\
x_{n+1}=\Pi_{C_{n+1}} x_{0}, \quad n \in \mathbb{N},
\end{array}\right.
$$

where $0<a \leq \beta_{n} \leq 1$, for all $n \in \mathbb{N}$. Then, $x_{n}$ converges strongly to $z_{0}=\Pi_{F(T)} x_{0}$.

Remark 3.3. Corollary 3.2 is equivalence to Theorem 4.1 of Takahashi, Takeuchi, Kubota [9]. Therefore, Our main result Theorem 3.1] is more general than Theorem 4.1 [9].

\section{Acknowledgement}

The authors thank the referee for the valuable comments and suggestions. And the first author is supported by Shandong Provincial Natural Science Foundation, China (2012ZRB01090); The second author is supported by the National Natural Science Foundation of China under grant(11071279).

\section{References}

[1] C. Chidume, Geometric properties of Banach spaces and nonlinear iterations, vol. 1965 of Lecture Notes in Mathematics, Springer, London, UK, 2009. 1 .

[2] R. Wittmann, Approximation of fixed points of nonexpansive mappings, Arch. Math. 58 (1992) 486-491. 1

[3] V. Berinde, Iterative Approximation of Fixed Points, vol. 1912 of Lecture Notes in Mathematics, Springer, Berlin, Germany, 2nd edition, 2007. 1

[4] K. Goebel, M.A. Pineda, On a type of generalized nonexpansiveness, , Proc. of the 8th International Conference on Fixed Point Theory and its Application 74 (2007) 660-665. 1, 1

[5] Klin-eam Chakkrid, Suantai Suthep, Fixed piont theorems for $\alpha$-nonexpansive mappings, Applied Mathematics Letters 23 (2010) 728-731. 1, 1.5, 1.6, $2,2.3$ 
[6] W. Takahashi, Nonlinear Functional Analysis. Fixed Points Theory and its Applications, okohama, Yokohama Publishers, 2000. 2

[7] I. Cioranescu, Geometry of Banach Spaces, Duality Mappings and Nonlinear Problems, Dordrecht, Kluwer Academic Publishers Group, 1990. 2

[8] Y.I. Alber, Metric and generalized projection operators in Banach spaces: properties and applications,in: Theory and Applications of Nonlinear Operators of Accretive and Monotone Type, Lecture Notes in Pure and Applied Mathematics, New York: Dekker (1996) 15-50. 2, 2.1, 2.2

[9] W. Takahashi, Y. Takeuchi, R. Kubota, Strong convergence theorems by hybrid methods for families of nonexpansive mappings in Hilbert spaces, J. Math. Anal. Appl. 341 (2008) 276-286. 3.3 